\title{
El aprendizaje autorregulado: presente y futuro de la investigación
}

\author{
Fermín Torrano Montalvo \\ María Carmen González Torres
}

Departamento de Educación, Universidad de Navarra

\section{España}

mctorrano@futurnet.es

\section{Resumen}

Los profundos cambios que ha experimentado el contexto de la Psicología de la Educación durante los últimos 30 años han llevado a que el aprendizaje autorregulado se convierta, actualmente, en tema central de la investigación y en uno de los ejes primordiales de la práctica educativa. A partir de la publicación de Zimmerman y Schunk (1989), Self-Regulated Learning and Academic Achievement: Theory, Research, and Practice, se han puesto en marcha un gran número de investigaciones sobre aprendizaje autorregulado. Tomando como referencia éstas y otras publicaciones actuales, el objetivo de este trabajo es recoger las principales cuestiones que se abordan dentro de las líneas de estudio del aprendizaje autorregulado. Además, se destacan una serie de direcciones que pueden guiar a las futuras investigaciones en este campo.

Palabras clave: aprendizaje autorregulado, estrategias de aprendizaje, motivación académica, rendimiento académico. 


\section{Introducción}

Los profundos cambios que ha experimentado el contexto de la Psicología de la Educación durante los últimos 30 años han conducido a que el aprendizaje autorregulado se convierta, actualmente, en tema central de la investigación y en uno de los ejes primordiales de la práctica educativa (Pintrich, 2000a; Reynolds y Miller, 2003). Actualmente, se concibe el aprendizaje como un proceso activo, cognitivo, constructivo, significativo, mediado y autorregulado (Beltrán, 1996).

El logro de un aprendizaje significativo y autorregulado necesita tanto "voluntad" (will) como "destreza” (skill) (Blumenfeld y Marx, 1997; McCombs y Marzano, 1990), y por esto, la educación debe ayudar a los alumnos a ser conscientes de su pensamiento, a ser estratégicos y a dirigir su motivación hacia metas valiosas. El objetivo es que los alumnos aprendan a ser sus propios maestros; y en este sentido se habla de la necesidad de pasar de la enseñanza a la práctica autorreflexiva (Schunk y Zimmerman, 1998).

A partir de la publicación en 1989 del libro Self-Regulated Learning and Academic Achievement: Theory, Research, and Practice (Zimmerman y Schunk, 1989), se han puesto en marcha un gran numero de investigaciones sobre aprendizaje autorregulado. Posteriormente, han ido apareciendo en la literatura otras publicaciones relevantes, en las cuales se recogen los principales avances en este campo:

- Self-Regulation of Learning and Performance (Schunk y Zimmerman, 1994).

- Self-Regulated Learning: From Teaching to Self-Reflective Practice (Schunk y Zimmerman, 1998).

- Handbook of Self-Regulation (Boekaerts, Pintrich y Zeidner, 2000).

- Self-Regulated Learning and Academic Achievement: Theoretical Perspectives (Zimmerman y Schunk, 2001).

Tomando como referencia éstas y otras publicaciones actuales, el objetivo de este trabajo es recoger las principales cuestiones que se abordan dentro de las líneas de estudio del aprendizaje autorregulado, destacando a su vez una serie de direcciones que pueden guiar a las futuras investigaciones en este campo. 


\section{Las características de los alumnos que autorregulan su aprendizaje}

De acuerdo con Zimmerman (2001, 2002), lo que caracteriza a los estudiantes autorregulados es su participación activa en el aprendizaje desde el punto de vista metacognitivo, motivacional y comportamental. Las características que se les atribuye a las personas autorreguladoras coinciden con las atribuidas a los alumnos de alto rendimiento y de alta capacidad, frente a los de bajo rendimiento (o con dificultades en el aprendizaje) que presentan déficit en esas variables (Reyero y Tourón, 2003; Roces y González Torres, 1998; Zimmerman, 1998). Sin embargo, con un adecuado entrenamiento en esas dimensiones, todos los estudiantes pueden mejorar su grado de control sobre el aprendizaje y el rendimiento, y se pueden paliar muchas de las dificultades de aprendizaje que presentan, particularmente, los sujetos de rendimiento bajo.

En general, los estudios señalan las siguientes características que diferencian a los alumnos que autorregulan su aprendizaje de los que no lo hacen (Corno, 2001; Weinstein, Husman y Dierking, 2000; Winne, 1995; Zimmerman, 1998, 2000, 2001, 2002):

1) Conocen y saben emplear una serie de estrategias cognitivas (de repetición, elaboración y organización), que les van ayudar a atender a, transformar, organizar, elaborar y recuperar la información.

2) Saben cómo planificar, controlar y dirigir sus procesos mentales hacia el logro de sus metas personales (metacognición).

3) Presentan un conjunto de creencias motivacionales y emociones adaptativas, tales como un alto sentido de autoeficacia académica, la adopción de metas de aprendizaje, el desarrollo de emociones positivas ante las tareas (p. ej., gozo, satisfacción, entusiasmo), así como la capacidad para controlarlas y modificarlas, ajustándolas a los requerimientos de la tarea y de la situación de aprendizaje concreta.

4) Planifican y controlan el tiempo y el esfuerzo que van a emplear en las tareas, y saben crear y estructurar ambientes favorables de aprendizaje, tales como buscar un lugar adecuado para estudiar y la búsqueda de ayuda académica (help-seeking) de los profesores y compañeros cuando tienen dificultades.

5) En la medida en la que el contexto lo permita, muestran mayores intentos por participar en el control y regulación de las tareas académicas, el clima y la estructura de la clase (p. ej., cómo será evaluado uno mismo, los requerimientos de las tareas, el diseño de los trabajos de clase, la organización de los grupos de trabajo). 
6) Son capaces de poner en marcha una serie de estrategias volitivas, orientadas a evitar las distracciones externas e internas, para mantener su concentración, su esfuerzo y su motivación durante la realización de las tareas académicas.

En pocas palabras, si hay algo que caracteriza a estos alumnos es que se sienten agentes de su conducta, creen que el aprendizaje es un proceso proactivo, están automotivados y usan las estrategias que les permiten lograr los resultados académicos deseados.

\section{Modelos sobre aprendizaje autorregulado}

En los últimos quince años se han propuesto numerosas teorías y modelos que han intentado identificar los procesos que intervienen en la autorregulación del aprendizaje, y establecer las relaciones e interacciones entre ellos y con el rendimiento académico. Puustinen y Pulkkinen (2001) han llevado a cabo una revisión de los modelos vigentes actualmente en este campo, analizando sus principales similitudes y diferencias. Dentro de todos ellos, estos autores destacan el modelo de Pintrich (2000b), como uno de los intentos de síntesis más importantes realizados sobre los diferentes procesos y actividades que ayudan a acrecentar la autorregulación del aprendizaje.

\section{El modelo de Pintrich}

Pintrich (2000b) ha propuesto un marco teórico, basado en una perspectiva sociocognitiva ${ }^{1}$, con el objetivo de clasificar y analizar los distintos procesos que, según la literatura científica, están implicados en el aprendizaje autorregulado. En dicho modelo, los procesos reguladores se organizan en función de cuatro fases: a) la planificación; b) la autoobservación (self-monitoring); c) el control; y d) la evaluación. A su vez, dentro de cada una de ellas, las actividades de autorregulación se enmarcan dentro de cuatro áreas: la cognitiva, la motivacional/afectiva, la comportamental y la contextual.

\footnotetext{
${ }^{1}$ La perspectiva socio-cognitiva del aprendizaje, nacida a la luz de los trabajos de Bandura (ver Bandura, 2001; Schunk, 2001, para una revisión), se caracteriza por estudiar la autorregulación como una interacción de procesos personales (cognitivos, motivacionales/afectivos y biológicos), comportamentales y contextuales.
} 
Tabla 1. Fases, áreas y procesos implicados en el aprendizaje autorregulado

(Tomado y traducido de Pintrich, 2000b, p. 454)

ÁREAS DE REGULACIÓN

\begin{tabular}{|c|c|c|c|c|}
\hline FASES & COGNICIÓN & $\begin{array}{l}\text { MOTIVACIÓN/ } \\
\text { AFECTO }\end{array}$ & $\begin{array}{c}\text { COMPORTAMIEN- } \\
\text { TO }\end{array}$ & CONTEXTO \\
\hline $\begin{array}{l}\text { 1. PREPARACIÓN } \\
\text { PLANIFICACIÓN } \\
\text { ACTIVACIÓN }\end{array}$ & $\begin{array}{l}\text { Estableci- } \\
\text { miento de } \\
\text { metas } \\
\text { Activación } \\
\text { del conoci- } \\
\text { miento pre- } \\
\text { vio } \\
\text { Activación } \\
\text { del conoci- } \\
\text { miento meta- } \\
\text { cognitivo }\end{array}$ & $\begin{array}{l}\text { Adopción de metas } \\
\text { Juicios de autoefica- } \\
\text { cia } \\
\text { Activación de las } \\
\text { creencias sobre el } \\
\text { valor de la tarea } \\
\text { Activación del } \\
\text { interés personal } \\
\text { Afectos (emociones) }\end{array}$ & $\begin{array}{l}\text { (Planificación del } \\
\text { tiempo y del es- } \\
\text { fuerzo) }\end{array}$ & $\begin{array}{l}\text { (Percepción de la } \\
\text { tarea) } \\
\text { (Percepción del con- } \\
\text { texto) }\end{array}$ \\
\hline $\begin{array}{l}\text { 2. AUTO- } \\
\text { OBSERVACIÓN } \\
\text { (Self-monitoring) }\end{array}$ & $\begin{array}{l}\text { Conciencia y } \\
\text { autoobserva- } \\
\text { ción de la } \\
\text { cognición }\end{array}$ & $\begin{array}{l}\text { Conciencia y auto- } \\
\text { observación de la } \\
\text { motivación y del } \\
\text { afecto }\end{array}$ & $\begin{array}{l}\text { Conciencia y } \\
\text { autoobservación } \\
\text { del esfuerzo, del } \\
\text { empleo del tiempo } \\
\text { y de la necesidad } \\
\text { de ayuda }\end{array}$ & $\begin{array}{l}\text { Conciencia y autoob- } \\
\text { servación de las con- } \\
\text { diciones de la tarea y } \\
\text { del contexto }\end{array}$ \\
\hline $\begin{array}{l}\text { 3. CONTROL } \\
\text { REGULACIÓN }\end{array}$ & $\begin{array}{l}\text { Uso de estra- } \\
\text { tegias cogni- } \\
\text { tivas y meta- } \\
\text { cognitivas }\end{array}$ & $\begin{array}{l}\text { Uso de estrategias de } \\
\text { control de la moti- } \\
\text { vación y del afecto }\end{array}$ & $\begin{array}{l}\text { Incremen- } \\
\text { to/disminución del } \\
\text { esfuerzo } \\
\text { Persistencia } \\
\text { Búsqueda de ayuda }\end{array}$ & $\begin{array}{l}\text { Cambios en los reque- } \\
\text { rimientos de la tarea y } \\
\text { en las condiciones del } \\
\text { contexto }\end{array}$ \\
\hline 4. EVALUACIÓN & $\begin{array}{l}\text { Juicios cogni- } \\
\text { tivos } \\
\text { Atribuciones }\end{array}$ & $\begin{array}{l}\text { Reacciones afectivas } \\
\text { Atribuciones }\end{array}$ & $\begin{array}{l}\text { Elección del com- } \\
\text { portamiento }\end{array}$ & $\begin{array}{l}\text { Evaluación de la tarea } \\
\text { y del } \\
\text { contexto }\end{array}$ \\
\hline
\end{tabular}

Estas cuatro fases representan para Pintrich una secuencia general por donde el alumno avanza a medida que realiza la tarea, pero no están jerárquica o linealmente estructuradas. Según este investigador, tales fases pueden darse de forma simultánea y dinámica, produciéndose una múltiple interacción entre los diferentes procesos y componentes incluidos en ellas. Asimismo, indica que no todas las tareas académicas implican explícitamente autorregulación: a veces, la realización de ciertas tareas no exige que el alumno planifique, controle y evalúe estratégicamente lo qué va a hacer, sino que, más bien, su ejecución se puede llevar a cabo de forma más o menos automática (o implícita) en función de la experiencia previa de los alumnos en las mismas. 
Como podemos observar en la tabla 1, los procesos autorreguladores arrancan en la fase de planificación, en la cual se destacan como actividades importantes: el establecimiento de las metas que se desean alcanzar o el objetivo específico que se quiere lograr con la tarea (target goal setting), la activación del conocimiento previo sobre la materia y del conocimiento metacognitivo (darse cuenta de las dificultades que entrañan las diferentes tareas, identificar los conocimientos y habilidades necesarias para abordarlas, conocimiento acerca de los recursos y estrategias que les pueden ayudar a solucionar la tarea, etc.) (área cognitiva); la activación de las creencias motivacionales (autoeficacia, metas, valor dado a la tarea, interés personal) y de las emociones (área motivacional/afectiva); la planificación del tiempo y del esfuerzo que se va a emplear en las tareas (área comportamental) y la activación de las percepciones respecto de la tarea y el contexto de clase (área contextual).

Dentro de la fase de autoobservación (self-monitoring) se incluyen las actividades que ayudan al alumno a tomar conciencia del estado de su cognición, su motivación, su afecto, su uso del tiempo y del esfuerzo, así como de las condiciones de la tarea y del contexto. Por ejemplo, aquí se incluyen aquellas relacionadas con la autoobservación de la comprensión (toma de conciencia metacognitiva). Éstas se manifiestan cuando los alumnos son conscientes de que no han entendido algo que acaban de leer o escuchar, cuando son conscientes de que están leyendo demasiado deprisa teniendo en cuenta la naturaleza del texto o sus metas (p. ej., comprender las ideas principales del mismo), o cuando observan activamente su comprensión lectora, haciéndose preguntas a sí mismos para saber si lo han entendido (Pressley y Afflerbach, 1995). Asimismo, esta fase abarca los procesos que los alumnos ponen en marcha para ser conscientes de su patrón motivacional (si se sienten competentes para realizar las tareas, si las valoran o qué metas guían y dirigen su conducta académica), de su propio comportamiento ("Tengo que dedicar más tiempo y esfuerzo para comprender este capítulo", "Necesito buscar ayuda"), así como de las características de la tareas y del contexto del aula (qué reglas existen en clase, cómo se evaluará el rendimiento, los requerimientos de la tarea, los sistemas de recompensas y castigos, la actuación del profesor, etc.).

Por otro lado, a la luz de los resultados de la fase anterior, se ponen en marcha las actividades de control, que engloban la selección y utilización de estrategias para controlar el pensamiento (uso de estrategias cognitivas y metacognitivas), la motivación y el afecto (estrategias motivacionales y de control emocional), así como las relacionadas con la regulación del 
tiempo y del esfuerzo y con el control de las diversas tareas académicas, el clima y la estructura de la clase.

En este punto, nos gustaría señalar que es muy difícil diferenciar la fase de autoobservación de la de control cognitivo, tal como aparece en algunos modelos de autorregulación (Butler y Winne, 1995; Nelson y Narens, 1990) en los que ambos aspectos se conciben como procesos separados. A pesar de que en un nivel conceptual sí que podrían diferenciarse los procesos implicados en la autoobservación y en el control de la cognición, los estudios empíricos realizados en esta área no apoyan dicha separación, ya que la mayoría de las veces ambos procesos ocurren simultáneamente (Pintrich, Wolters y Baxter, 2000).

Finalmente, la fase de reflexión o evaluación abarca los juicios y las evaluaciones que los alumnos realizan acerca de su ejecución en la tarea, comparándola con los criterios previamente establecidos por él (o por el profesor); las atribuciones que realizan sobre las causas de sus éxitos o fracasos; las reacciones afectivas que experimentan ante los resultados, como consecuencia de las atribuciones realizadas; la elección del comportamiento que se llevará a cabo en el futuro, así como las evaluaciones generales sobre la tarea y el ambiente de clase.

En resumen, el modelo de Pintrich se ofrece como un marco global y comprensivo desde el que poder analizar pormenorizadamente los distintos procesos cognitivos, motivacionales/afectivos, comportamentales y contextuales que promueven el aprendizaje autorregulado. Una de las novedades presentes en este modelo frente a otras es que por primera vez se incluye, como área sujeta a autorregulación, el área contextual. En consonancia con los nuevos modelos de la enseñanza basados en una perspectiva socioconstructivista, tales como las comunidades de aprendizaje (Brown, 1997; Brown y Campione, 1990) y la escuela centrada en el aprendiz (learner-centered classroom) (McCombs y Whisler, 2000), en este modelo se destaca que los estudiantes sí que pueden hacer algo por cambiar y modificar su contexto, por lo que este aspecto debe considerarse como una cuestión importante en la autorregulación del aprendizaje.

\section{El papel de la motivación académica en la autorregulación del aprendizaje}

Si durante los primeros años del boom de la revolución cognitiva en la Psicología de la Educación (años 70-80), los estudios centraron su atención en el papel de las variables cogni- 
tivas (estudios sobre el procesamiento de la información, estilos cognitivos, estrategias de aprendizaje, conocimiento previo, procesos de pensamiento), en la década de los ochenta y noventa la investigación en este campo recibió un gran impulso cuando confluyeron en su mismo camino las investigaciones realizadas en el campo motivacional (estudios sobre autoconcepto, creencias de autoeficacia, atribuciones, metas, etc.). Esto incrementó el interés por estudiar cómo las variables mencionadas se entrelazan e influyen en los resultados de aprendizaje, lo que dio lugar al desarrollo de la investigación sobre aprendizaje autorregulado (González Torres y Tourón, 1992; Pintrich, 2003; Roces y González Torres, 1998).

Los estudios realizados en este campo coinciden en que la intervención en estrategias de aprendizaje favorece el aprendizaje cognitivo y la motivación para aprender, y, por otra parte, que la mejora de las creencias motivacionales de los estudiantes no sólo influyen en la motivación para aprender, sino también en el modo y en la calidad con que los alumnos procesan la información, seleccionando y usando determinadas estrategias de aprendizaje.

En concreto, los estudios sobre aprendizaje autorregulado realizados en los últimos años, recogiendo las aportaciones de las teorías cognitivas de la motivación, que arrancan del modelo de expectativa x valor de Atkinson (cfr. Wigfield y Eccles, 2000), destacan la importancia de las expectativas de autoeficacia (convicción que tiene el individuo de poder realizar con éxito la conducta que se requiere para producir unos resultados determinados) (Bandura, 1977) y de las metas (razones para realizar las tareas), como aspectos críticos de la motivación que influyen en el control y regulación del aprendizaje.

Las creencias de autoeficacia, de las que inicialmente se ocupó Bandura $(1977,1986)$ en sus estudios sobre la autorregulación de la conducta, no son ni rasgos globales de la personalidad ni tampoco autoconceptos generales, sino más bien autoconcepciones específicas que los individuos elaboran principalmente a partir de sus experiencias de dominio (éxitos/fracasos) en distintas actividades. Bandura (1997) considera que las creencias de autoeficacia, al caracterizarse por su especificidad situacional y conductual, son mejores predictores de la motivación y de la conducta en un campo concreto que los índices globales del autoconcepto. Es por ello que este constructo ha recibido una gran atención en la investigación motivacional y en el campo del aprendizaje autorregulado. 
A este respecto, son destacables las investigaciones realizadas por Pintrich y sus colaboradores con el MSLQ (Motivated Strategies Learning Questionnaire) (Pintrich, 2003; Pintrich, Smith, García y McKeachie, 1991; Roces, 1996), de naturaleza correlacional ${ }^{2}$ y con muestras de alumnos de secundaria y de universidad, y los estudios de Zimmerman y Schunk (cfr. Schunk, 2001; Schunk y Ertmer, 2000; Zimmerman, 2001, para una revisión), que han destacado el positivo efecto de las creencias de autoeficacia académica en todo el proceso de la autorregulación.

Una cuestión de gran interés para la investigación en esta área está siendo dilucidar las diferencias que existen entre las creencias de autoeficacia y el autoconcepto. Y es que en la investigación ha habido una tendencia a desarrollar medidas de autoeficacia general, muy similares a las de autoconcepto desarrolladas a partir del modelo de Shavelson, Hubner y Stanton (1976), lo cual representa un problema, pues al perder su especificidad situacional disminuye la capacidad predictiva de estas creencias. Así, en los últimos años, diversas investigaciones, como las de Bong y Clark (1999), Bong y Skaalvik (2003), y Pajares y Schunk (2001), han tratado de afinar más sutilmente las características diferenciales entre ambos constructos.

Resumidamente, ya que nuestro objetivo no es el de profundizar en las diferencias y similitudes que existen entre tales autocreencias (para ello, remitimos al lector a los trabajos reseñados anteriormente), nos gustaría señalar que, a pesar del excesivo afán de los investigadores por separar autoconcepto y autoeficacia, si nos fijamos detenidamente en los aspectos que las configuran, ambas creencias comparten características muy parecidas en cuanto a su naturaleza multidimensional (Bong, 1998, Marsh, 1990), en las fuentes que utilizan los alumnos para su creación y desarrollo (cfr. Bong y Clark, 1999; Skaalvik, 1997), y, cuando se toman medidas específicas de ambos constructos, en la relación que mantienen con el rendimiento académico (Bong y Clark, 1999; Pajares y Schunk, 2001; Skaalvik, 1997).

Todas estas razones nos llevan a pensar, en consonancia con Bong y Skaalvik (2003), que tal vez la distinción tan notable que se quiere hacer entre dichas creencias puede estar sobrestimada, y que las diferencias tan sobresalientes que los investigadores han querido

2. En nuestro contexto, González Pienda, Núñez y Roces, de la Universidad de Oviedo, y González Cabanach, Valle y Rodríguez, de la Universidad de la Coruña, han llevado a cabo una serie de investigaciones con el objetivo de determinar la relación causal entre la motivación, las estrategias de aprendizaje y el rendimiento académico (cfr. Rodríguez, 1999; Valle, González Cabanach, Vieiro y Suárez, 1998). 
hacer entre estos constructos no han hecho más que aumentar la confusión a la hora de utilizarlos. En determinadas situaciones, como cuando medimos el autoconcepto en sus dimensiones específicas y las creencias de autoeficacia en un nivel general, ambas creencias son empíricamente similares. En otras palabras, que las diferencias entre ambos constructos pueden ser superadas fácilmente.

Por otro lado, con respecto a la investigación sobre metas académicas, desarrollada a partir de las teorías de Dweck (1986) y Nicholls (1984), la mayoría de los estudios se han centrado en estudiar el impacto de dos tipos de metas en el aprendizaje autorregulado: las metas de aprendizaje ${ }^{3}$ (mastery, task, learning goals) y las metas de rendimiento ${ }^{4}$ (performance, ego, ability goals).

Numerosos estudios muestran que los alumnos que persiguen y adoptan metas de aprendizaje utilizan más estrategias cognitivas profundas (de elaboración y organización) y metacognitivas (actividades de planificación de metas y de autoobservación de la propia comprensión), tienen unas creencias motivacionales más adaptativas hacia sí mismos y hacia las tareas (altas creencias de autoeficacia ante tareas difíciles, la formación de un patrón atribucional adaptativo, un gran interés intrínseco y disfrute en las tareas, altos niveles de valor, utilidad e importancia asignados a las mismas, un mayor número de reacciones afectivas positivas ante las tareas), y presentan mayores niveles de esfuerzo y persistencia, así como más conductas relacionadas con la búsqueda de ayuda académica cuando tienen dificultades, que los alumnos con otros tipos de metas (cfr. Kaplan, Middleton, Urdan y Midgley, 2002; Pintrich, 2000b, para una revisión).

En el caso de las metas de rendimiento, sin embargo, nos gustaría señalar que existe una mayor discrepancia sobre su repercusión en la motivación y en el aprendizaje autorregulado. Y es que en muchos estudios los hallazgos obtenidos han sido bastante dispares e, incluso, contradictorios entre sí. Si los primeros trabajos realizados en esta área mostraron que estas metas estaban asociadas a un conjunto de factores cognitivos (uso de estrategias superficiales), motivacionales/afectivos (atribuciones del fracaso a la baja capacidad, bajo interés por

3. Los alumnos orientados hacia metas de aprendizaje se caracterizan por estar centrados en el proceso de aprendizaje y por el deseo de desarrollar sus capacidades y ampliar su comprensión a la hora de realizar las tareas (Urdan, 1997).

4. Los alumnos que persiguen metas de rendimiento están orientados a demostrar competencia y a intentar ser mejor que los demás (Urdan, 1997). 
la tarea, alta ansiedad ante los exámenes) y comportamentales (uso de estrategias selfhandicapping) perjudiciales para el aprendizaje, no obstante, en dichos estudios no se distinguieron empíricamente los componentes de aproximación y evitación de tales metas (approach and avoidance motives), por lo que no se pudo llegar a una única integración de los resultados (Elliot, 1999).

Actualmente, con la nueva reconceptualización de la teoría de metas llevada a cabo por Pintrich, Barron, Elliot, Harackiewicz y sus colaboradores en la segunda mitad de la década de los noventa, y en la que distinguen tales componentes, se ha puesto de relieve que los efectos de las metas de rendimiento en la motivación y en el rendimiento son diferentes, según predomine el objetivo de aproximarse a la tarea con el fin de demostrar la propia competencia (performance-approach) o con el de evitar juicios negativos sobre la propia valía (performance-avoidance) (Harackiewicz, Barron, Pintrich, Elliot y Thrash, 2002).

En general, los hallazgos apuntan a que los alumnos orientados a demostrar competencia (performance-approach) informan de aspectos positivos en su motivación (aumento de sus creencias de autoeficacia y de su interés e implicación en las tareas cuando tienen éxito en sus objetivos) y en su cognición (cierto uso de estrategias cognitivas y metacognitivas), aunque los hallazgos encontrados respecto a esta última cuestión son bastante contradictorios entre sí (cfr. Barron y Harackiewicz, 2000; Harackiewicz et al., 2002; Midgley, Middleton y Kaplan, 2001; Pintrich, 2000b). Sin embargo, a pesar de tales beneficios, se ha constatado que el hecho de centrarse en demostrar competencia y en compararse con los demás puede tener algunos costes, como por ejemplo, la evitación de tareas difíciles, un aumento de la ansiedad ante los exámenes, así como una disminución en el empleo de determinadas estrategias de autorregulación, como la búsqueda de ayuda académica (Newman, 1998; Pintrich, 2000b; Urdan, Ryan, Anderman y Gheen, 2002).

Por otra parte, los estudios coinciden en señalar que el componente de evitación de las metas de rendimiento (performance-avoidance) no es la mejor orientación para implicarse en las tareas académicas. Como se ha demostrado, los alumnos con estas metas muestran un patrón motivacional, afectivo, cognitivo y comportamental muy perjudicial para el aprendizaje y la motivación (Pintrich, 2000b). Por ejemplo, suelen hacer uso de estrategias selfhandicapping con el objetivo de proteger sus sentimientos de autovalía ante situaciones de fracaso; no se esfuerzan en el uso de estrategias cognitivas y metacognitivas y se limitan a 
cumplir exclusivamente con los requerimientos de la tarea; atribuyen los fracasos a factores internos y estables, como la falta de capacidad; tienen bajas creencias de autoeficacia; presentan un interés y un valor por la tarea negativo; experimentan una gran ansiedad ante los exámenes y el rendimiento académico; y muestran bajos niveles de esfuerzo y persistencia en las tareas $^{5}$, así como de conductas relacionadas con la búsqueda de ayuda académica (Dweck, 2002; Middleton y Midgley, 1997; Midgley y Urdan, 2001; Ryan y Pintrich, 1997; Wolters, 2003).

En síntesis, nos gustaría señalar que, actualmente, en las nuevas reconceptualizaciones de la teoría de metas se está superando la tendencia a considerar que las metas de rendimiento son inadecuadas para promover una motivación óptima y el deseo de aprender. De hecho, como ya señaló Dweck (1986), los alumnos únicamente interesados en metas de aprendizaje, pero no en alcanzar otros objetivos (sacar buenas notas, hacer las tareas que se mandan en clase, lograr los objetivos de clase), pueden estar actuando contra sus intereses e, incluso, pueden poner en riesgo oportunidades para su futuro aprendizaje (acceso a determinadas carreras universitarias, salida profesional, etc.).

Esta nueva perspectiva en el estudio de metas critica que muchos estudios se han centrado exclusivamente en examinar por separado los efectos de las diferentes metas, sin prestar apenas atención a los efectos positivos que puede comportar perseguir múltiples metas al mismo tiempo. Así, se sugiere que para tener éxito en la escuela, los alumnos deben orientarse tanto a metas intrínsecas (ampliar conocimientos, dominar la tarea, desarrollar capacidades, etc.) como extrínsecas (p. ej., intentar obtener buenas notas, rendir más que los demás y obtener juicios positivos de ello, perseguir metas relacionadas con la responsabilidad social, etc.) (cfr. Barron y Harackiewicz, 2000; González Torres, 1997; Harackiewicz et al., 2002; Lin, McKeachie y Kim, 2003; Pintrich, 2000c; Valle et al., 2003; Wentzel, 2000).

Por otra parte, la investigación centrada en el estudio de metas desde una perspectiva social ha puesto de relieve que una orientación hacia metas sociales, sobre todo hacia aquellas relacionadas con la responsabilidad social, en coordinación con una orientación hacia metas académicas (aprendizaje/aproximación al rendimiento), es una de las maneras más viables y

5. Estos alumnos, al estar centrados en no aparecer como incompetentes ante los demás, pueden realizar algún tipo de esfuerzo con este fin (p. ej., estudiar para evitar sacar las peores notas), pero no de la misma manera que los alumnos con metas de aprendizaje o que intentan demostrar competencia. 
beneficiosas de aumentar el aprendizaje y el rendimiento (Patrick, Anderman y Ryan, 2002; Urdan y Maehr, 1995; Wentzel, 2000, 2002, 2003).

\section{La evaluación del aprendizaje autorregulado}

Una cuestión de gran importancia y difícil para la investigación está relacionada con la medida o estimación de los diferentes componentes y procesos del aprendizaje autorregulado (Schraw y Impara, 2000; Winne, Jamieson-Noel y Muis, 2002; Winne y Perry, 2000).

En un intento por clarificar y clasificar los métodos e instrumentos utilizados por los investigadores para medir los procesos implicados en la autorregulación del aprendizaje, Winne y Perry (2000) distinguen entre: a) instrumentos que miden el aprendizaje autorregulado como una aptitud, los cuales describen alguna de las cualidades o atributos relativamente estables del alumno que autorregula su aprendizaje, y que permiten predecir su conducta (cognición y motivación) futura; y b) instrumentos que miden el aprendizaje autorregulado como una actividad (event), caracterizados por ser medidas más complejas que recogen información sobre los estados y procesos que el alumno despliega a lo largo del tiempo mientras se autorregula.

Dentro de la primera categoría, se incluyen los cuestionarios de autoinformes, las entrevistas estructuradas y los juicios de los profesores, y, dentro de la segunda, los protocolos think-aloud, los métodos de detección de errores en las tareas, las trace methodologies y las medidas de observación.

\section{a) Instrumentos de medida del aprendizaje autorregulado como aptitud:}

\section{Los cuestionarios de autoinforme}

Hasta la fecha, son los procedimientos más utilizados para medir el aprendizaje autorregulado, debido a su facilidad para el diseño, administración e interpretación de los resultados. Estas medidas están basadas en el autoinforme que el propio sujeto ofrece. Algunos de los cuestionarios más utilizados son:

- The Learning and Study Strategies Inventory (LASSI) (Weinstein, Schulte y Palmer, 1987). El LASSI es un cuestionario de autoinforme de 77 ítems, 
diseñado con el objetivo de valorar las estrategias de aprendizaje que utilizan los alumnos universitarios. Los ítems de la versión de 1987 están agrupados en 10 escalas: actitud, motivación, organización del tiempo, ansiedad, concentración, procesamiento de la información, selección de ideas principales, uso de técnicas y materiales de apoyo, autovaloración y estrategias de examen. En nuestro contexto ha sido una de las escalas más utilizadas para medir estrategias de aprendizaje (ver, p. ej., Durán, 1999; González Pienda, Núñez, Rodríguez y González Cabanach, 1994; Prieto y Castejón, 1993). Sin embargo, se ha constatado la falta de una adecuada validez de constructo de esta escala y la necesidad de realizar un examen y una revisión de la misma antes de emplearse en investigaciones futuras.

- $\quad$ The Motivated Strategies for Learning Questionnaire (MSLQ) (Pintrich et al., 1991). Pintrich y sus colaboradores han creado este autoinforme de 81 ítems, basado en el modelo motivacional de expectativa x valor (Pintrich, 2003; Wigfield y Eccles, 2000), con el objetivo de medir diferentes componentes motivacionales y el uso de estrategias de aprendizaje en un curso o en una materia específica (ver tabla 2). Una de las ventajas de este instrumento es que se ha aplicado y validado en distintos niveles educativos, tanto universitarios como no universitarios. En nuestro contexto, este cuestionario ha sido traducido y adaptado por Roces, Tourón y González Torres (1995), y dado a conocer con el nombre de CEAM II (Cuestionario de Estrategias de Aprendizaje y Motivación).

Tabla 2. Escalas y subescalas del MSLQ

\begin{tabular}{ccc}
\hline ESCALAS & DIMENSIONES & SUBESCALAS \\
\hline & Componentes de expec- & Creencias de control \\
tativas & Autoeficacia \\
MOTIVACIÓN & Componentes de valor & Metas intrínsecas \\
& & Malor de la tarea \\
& & \\
& Componentes afectivos & Ansiedad en los exámenes
\end{tabular}




\begin{tabular}{ccc}
\hline ESCALAS & DIMENSIONES & SUBESCALAS \\
\hline & & Repetición \\
& Estrategias cognitivas y & Elaboración \\
metacognitivas & Organización \\
APRENDIZAJE & & Pensamiento crítico \\
& & Metacognición \\
& & Tiempo y lugar de estudio \\
& Estrategias de manejo de & Regulación del esfuerzo \\
& recursos & Aprendizaje con "otros" \\
& & Búsqueda de ayuda \\
\hline
\end{tabular}

- The Components of Self-Regulated Learning (CSRL) (Niemivirta, 1998). Niemivirta (1998) ha elaborado este cuestionario con el objetivo de medir los componentes motivacionales y cognitivos implicados en el aprendizaje autorregulado. Por un lado, con relación a la motivación académica, en el CSRL se valoran los constructos de metas, creencias de control y el de autoestima. Por otro lado, respecto a la cognición, se valora el uso que hacen los alumnos de las estrategias de aprendizaje y de autorregulación. Aquí se incluyen diferentes estrategias asociadas a distintos niveles de procesamiento de la información: desde un nivel superficial, en el que se engloban las estrategias de memorización del material, hasta un nivel profundo, el cual abarca las estrategias de elaboración, planificación de metas y autoobservación de la propia comprensión.

\section{Las entrevistas estructuradas}

Uno de los procedimientos de entrevista más utilizado para medir aprendizaje autorregulado ha sido la Self-Regulated Learning Interview Schedule (SRLIS) (Zimmerman y Martínez-Pons, 1986, 1988). Zimmerman y sus colaboradores, a partir de la identificación de catorce tipos de estrategias que los alumnos de secundaria utilizaban dentro y fuera de clase para autorregular su aprendizaje, desarrollaron un procedimiento de entrevista estructurada para valorarlas.

Estudios posteriores han confirmado la validez de este procedimiento para medir el uso de las estrategias de autorregulación y para discriminar entre los estudiantes de alto y bajo 
rendimiento según el uso que hacen de ellas. Las catorce estrategias medidas por este procedimiento son: organización y transformación de la información, autoevaluación, establecimiento de metas y planificación, búsqueda de información, registro y control, estructuración del ambiente, autoasignación de sanciones positivas y negativas (self-consequences), repetición y memorización, búsqueda de ayuda de iguales, búsqueda de ayuda de profesores, búsqueda de ayuda de adultos y revisión o repaso de los exámenes, apuntes y libros de texto.

\section{Los juicios de los profesores}

En este caso, los profesores son los que evalúan la calidad del aprendizaje autorregulado de los alumnos a través de las actividades académicas diarias. Zimmerman y MartínezPons (1988) han creado una escala para el profesor, denominada Rating Student Selfregulated Learning Outcomes: A Teacher Scale, con el objetivo de que los profesores midan el uso que hacen los alumnos de estrategias de autorregulación. A través de un cuestionario de 12 ítems, los profesores puntúan en una escala tipo Lickert de 5 puntos si el alumno emplea alguna de las catorce estrategias identificadas en el SRLIS.

\section{b) Instrumentos de medida del aprendizaje autorregulado como actividad:}

\section{Las medidas think-aloud}

El think-aloud (pensar en voz alta) es un protocolo en el que el alumno informa de sus pensamientos y de los procesos y estrategias cognitivas que pone en marcha durante la realización de la tarea. Una de las áreas donde más se ha utilizado este protocolo ha sido en la lectura (Pressley, 2000; Pressley y Afflerbach, 1995). Asimismo, hay que señalar que las respuestas verbales del alumno se analizan empleando el protocolo de respuesta utilizado por Zimmerman y Martínez-Pons (1986).

\section{Los métodos de detección de errores en las tareas}

Estos instrumentos suelen emplearse habitualmente para evaluar el proceso de autoobservación de la comprensión en el área de lectura. Para ello, los investigadores introducen algunos errores dentro de los materiales que los estudiantes utilizan para estudiar (p. ej., en 
textos) con el fin de observar si los errores son detectados y qué es lo que hacen cuando los descubren (Baker y Cerro, 2000; Garner, 1987).

\section{Trace Methodologies}

Estos instrumentos se basan en señales o indicadores observables sobre los procesos cognitivos que los alumnos despliegan mientras realizan las tareas. Por ejemplo, uno de los indicadores que se ha empleado para medir el proceso de control cognitivo se refiere a si el alumno escribe más información de la necesaria en los márgenes de su cuaderno o de su libro escolar, como notas al pie, resúmenes, comentarios personales, diagramas, confrontación de la información con otras fuentes, etc. (Baker y Cerro, 2000; Winne y Jamieson-Noel, 2003).

\section{Medidas de observación de la ejecución en la tarea}

Estas medidas se basan en la observación por parte de jueces de lo que hacen los alumnos mientras realizan las tareas (ver, p. ej., Perry, 1998). Frecuentemente, suelen ser complementadas con entrevistas. Las ventajas de estas medidas en la evaluación del aprendizaje autorregulado son varias (cfr. Turner, 1995): son medidas objetivas de lo que hacen los aprendices en vez de lo que recuerdan o creen hacer; permiten relacionar las conductas de los alumnos con las condiciones que requieren las tareas; $y$, finalmente, pueden disminuir las dificultades asociadas con la medición de este proceso en niños, como por ejemplo, con el sesgo de respuesta ante los cuestionarios (tienden a contestar de manera muy optimista) y con las limitaciones de estos sujetos para describir los procesos cognitivos que ponen en marcha durante la realización de la tareas.

\section{La enseñanza del aprendizaje autorregulado}

En el libro Self-Regulated Learning: From Teaching to Self-reflective Practice (Schunk y Zimmerman, 1998) se pueden encontrar diferentes ejemplos de intervenciones y modelos instruccionales diseñados con el objetivo de enseñar los procesos y las estrategias involucradas en el aprendizaje autorregulado (ver también García Ros, Clemente y Pérez González, 1994; González Torres, 1997; Monereo y Castelló, 1997; Sanz de Acedo e Iriarte, 1999, para una revisión). 
Los puntos comunes (o estrategias didácticas) en los que coinciden directamente las distintas intervenciones y programas son éstos: la enseñanza directa de estrategias, el modelado, la práctica guiada y autónoma de las estrategias, la retroalimentación, la autoobservación, el apoyo social y su retiro en el momento en que el estudiante ha alcanzado cierto grado de participación responsable, y la autorreflexión.

Con la enseñanza directa de estrategias se explica al alumno qué y cuáles son las características de las estrategias que les pueden ayudar a procesar mejor la información y a regular su aprendizaje, cómo se utilizan y qué habilidades están implicadas en ellas, cuándo, cómo y porqué hay que utilizar unas u otras estrategia, y para qué sirven, es decir, cuáles son sus beneficios.

El modelado es uno de los procedimientos más indicados para la enseñanza de las estrategias de autorregulación (Graham, Harris y Troia, 1998). La acción de planificar, controlar la ejecución, distribuir los recursos cognitivos y reflexionar sobre lo realizado, podrían ser asimilados por los alumnos, observando cómo la lleva a cabo el docente u otros modelos expertos (peer model) (Schunk y Zimmerman, 1998, 2003).

La práctica, primero guiada y luego independiente, de las estrategias de autorregulación, y el suministro de feedback por parte de los "otros" (principalmente del profesor) sobre la efectividad de las estrategias, son procedimientos que mejoran el aprendizaje y la motivación de los alumnos, ya que promueven la transferencia de estrategias y su mantenimiento. El objetivo de la práctica guiada y autónoma es que la responsabilidad o el control para generar, aplicar y evaluar las estrategias sea transferido del profesor al alumno (Onrubia, 1996). Este tipo de aprendizaje mediado, propuesto inicialmente por Vygotsky y otros autores (p. ej., Feuerstein), es uno de los componentes esenciales de muchos modelos y programas instruccionales (p. ej., andamiaje, enseñanza recíproca, etc.) (Valle, González Cabanach, Vieiro y Suárez, 1998).

La autoobservación (self-monitoring) es un componente muy importante en la intervención, ya que si el alumno desea aprender estrategias, de algún modo tiene que supervisar la aplicación de las mismas, su efectividad y la manera de cambiarlas o modificarlas en el caso de que hayan sido ineficaces. 
El suministro de apoyo social al alumno por parte de los profesores y de los compañeros a la vez que aprende las estrategias de autorregulación es también una de las estrategias más utilizadas por los diversos programas. Unido a esto se encuentra la supresión del apoyo a medida que el estudiante sea más competente en su adquisición y desarrollo. A este respecto, Graham et al. (1998) afirman que la retirada de este apoyo o andamiaje (scaffolding) se ha de hacer de forma escalonada, pasando de la mediación más directiva e intensa en la etapa inicial a formas más autorreguladas.

Finalmente, el proceso didáctico que siguen todos estos programas culmina en la práctica autorreflexiva (o discusión metacognitiva), donde los alumnos practican de modo independiente las habilidades y estrategias adquiridas, reflexionan sobre el proceso de aprendizaje que se ha seguido, evalúan el rendimiento obtenido y la efectividad de las estrategias, modifican, en el caso de que sea necesario, la perspectiva utilizada, y realizan ajustes en su entorno social y físico para crear un ambiente más favorable para su aprendizaje.

Como señalan Paris y Paris (2001, p. 91), en los últimos treinta años la naturaleza de la instrucción en este campo ha cambiado de forma drástica. Si en un primer momento (años 70-80), se acentuaba la enseñanza explícita de estrategias (instrucción directa), actualmente, los modelos instruccionales ponen el acento en la práctica autorreflexiva y en la instrucción por andamiaje (scaffolding instruction), puesto que el objetivo central es la metacognición, es decir, el desarrollo de la consciencia y control de nuestros procesos de reflexión. Por otra parte, también se observa en el área de la intervención una tendencia a integrar la enseñanza de estrategias dentro de tareas y materias curriculares específicas.

Ley y Young (2001; ver también Paris y Paris, 2001), basándose en los estudios que apoyan la influencia positiva de la autorregulación en el aprendizaje y en el rendimiento y que identifican los déficit de los alumnos que no regulan su aprendizaje (p. ej., Zimmerman, 1998), proponen una serie de pautas generales para el diseño de la instrucción, orientada a ayudar a los alumnos -menos expertos en autorregulación- a ser más estratégicos y autorregulados. Concretamente, según estos autores habría que:

- Ayudar a los alumnos a crear y estructurar ambientes favorables de aprendizaje. Concretamente, esto se podría llevar a cabo ayudándoles a desarrollar estrategias volitivas dirigidas a evitar las distracciones internas y del entorno (ruidos, molestias de los compañe- 
ros, etc.), y eliminarlas o disminuirlas, manteniendo su atención y su esfuerzo en la tarea que están llevando a cabo. A este respecto, Corno $^{6}(1993,2001)$ ha propuesto seis tipos de estrategias fundamentales que los estudiantes pueden poner en marcha para permanecer centrados en la tarea y llevar a término sus intenciones de aprendizaje: a) estrategias volitivas o de control encubiertas (covert volitional control), destinadas a controlar el mundo interno del sujeto (cognición, motivación, emoción); y b) estrategias volitivas de control externo o descubiertas (overt volitional control), dirigidas al control de los aspectos externos al sujeto en relación con la tarea y el contexto.

- Organizar la instrucción y las actividades de modo que se facilite el uso de estrategias cognitivas y metacognitivas.

- Dotar al alumno de oportunidades para que autoobserve (self-monitoring) su aprendizaje. Este proceso, parte nuclear de la autorregulación (Butler y Winne, 1995; Zimmerman, 1998), depende a su vez de otros dos procesos: el establecimiento de metas y el feedback suministrado por los demás y por uno mismo (habla interna). Por tanto, en este caso se puede animar a los alumnos a que autoobserven su aprendizaje: por un lado, ayudándoles a que utilicen el feedback interno y externo para supervisar su grado de cumplimiento respecto a las metas propuestas, y si son efectivas o no las estrategias que están utilizando; y, por otro, haciéndoles ver la importancia de establecer metas próximas, realistas y específicas, ya que de esta manera los progresos y los avances son más fáciles de constatar. Asimismo, el alumno puede estimular la autoobservación, registrando aspectos relacionados con las tareas académicas (p. ej., tiempo empleado en finalizarlas, en tomar notas, en leer el texto, etc.), ya que estas actividades facilitan la generación de feedback, que puede guiar los esfuerzos para conseguir las metas futuras.

- Proporcionar a los estudiantes información evaluativa continua y darles ocasión para autoevaluar su aprendizaje. Se debe recalcar el nivel logrado en los objetivos, si se mejoraron los conocimientos sobre el tema y la efectividad de las estrategias que se están utilizando.

6. Desde hace algunos años, se aprecia dentro de la Psicología un movimiento de recuperación de la voluntad para explicar el paso de la intención a la acción, y que ya está siendo recogido en los estudios sobre la autorregulación del aprendizaje. El actual despertar del interés por ella es atribuible a Kuhl (2000) y Heckhausen (1991) psicólogos alemanes, y en el campo educativo a la investigadora americana Corno (2001). Estos autores, complementando los tradicionales enfoques de la motivación y de la autorregulación, que se centran en el rol mediador de las creencias (expectativas, valores, metas), están estudiando, desde una perspectiva más funcional, los factores y las fuerzas dinámicas relacionadas con la voluntad, que parecen necesarias para mover a los individuos hacia las metas que se proponen (González Torres, 2003). 
Asimismo, es muy importante proporcionar a los alumnos feedback correctivo que les ayude a ver donde estaban equivocados y cómo corregir los problemas.

En nuestro contexto, los profesores e investigadores interesados en la instrucción para fomentar el aprendizaje autorregulado pueden encontrar diversas propuestas instruccionales en los programas de De la Fuente y Martínez Vicente (Programa Prorregula, 2000), Yuste y Ayala (Progresint 31, 2000), y Hernández y García (Notice, 1997), entre otros.

\section{Futuras direcciones en la investigación}

Diversas publicaciones en este campo (cfr. Butler, 2002; Eccles, Wigfield y Schiefele, 1998; Paris y Paris, 2001; Patrick y Middleton, 2002; Pintrich, 2003; Pintrich et al., 2000; Schunk y Zimmerman, 2003; Zeidner, Boekaerts y Pintrich, 2000) apuntan algunas de las principales direcciones en las que deberían centrarse las futuras investigaciones:

1) La mejora en la definición y operativización de los principales procesos y actividades involucradas en el aprendizaje autorregulado, y las diferencias existentes entre este constructo y los relacionados con él (p. ej., autocontrol, metacognición).

2) La elaboración de modelos más completos, que incorporen conceptos referidos a las fuerzas dinámicas que afectan al proceso de la autorregulación (p. ej., procesos volitivos).

3) El perfeccionamiento de la metodología de la investigación y de los instrumentos de medida. Se hace necesario utilizar, por un lado, diseños más complejos (p. ej., diseños longitudinales, estudios causales y no sólo correlacionales) y, por otro, crear y validar un mayor número de métodos e instrumentos de naturaleza cualitativa, que se complementen y se contrasten con el uso de autoinformes, ya que permitirán a los investigadores investigar el aprendizaje autorregulado como un proceso dinámico y continuo (event), que se despliega a lo largo del tiempo y en un contexto específico, y superar las limitaciones asociadas al uso exclusivo de autoinformes en su valoración. Asimismo, Pintrich (2003), desde una perspectiva motivacional, plantea el interés de llevar a cabo análisis experimentales clásicos, al igual que hacen los psicólogos puramente cognitivos, con el objetivo de examinar en profundidad los efectos de la motivación en la cognición y en el aprendizaje. 
4) El análisis del papel del contexto de aprendizaje sobre la cognición y la motivación académica. La investigación realizada en los últimos años desde una perspectiva socioconstructivista (p. ej., McCaslin y Hickey, 2001; Paris, Byrnes y Paris, 2001; Volet y Jarvela, 2001) está mostrando cómo las características del contexto y las demandas de la situación afectan al aprendizaje y a la motivación de los estudiantes. Por eso, es necesario avanzar en el desarrollo de modelos de enseñanza centrados en el aprendiz y en las llamadas comunidades de aprendizaje. En estos modelos, y basándose en la teoría de la autodeterminación de Deci y Ryan (2002), se destaca la importancia de estructurar el contexto de modo que se satisfagan las necesidades de competencia, autonomía y conexión afectiva de los alumnos, y así, favorecer la automotivación y el aprendizaje autorregulado. Por otro lado, el modelo TARGET de Ames (1992), cuyos trabajos han sido continuados posteriormente por Midgley y sus colaboradores (cfr. Midgley, 2002), aporta sugerencias interesantes para diseñar ambientes de aprendizaje, orientados al desarrollo del aprendizaje autorregulado y la motivación para aprender. Finalmente, y respondiendo a los nuevos cambios que está experimentando la escuela (p. ej., la introducción de las tecnologías de la información y de la comunicación en el aula), se debe estudiar cómo influyen estos nuevos ambientes de aprendizaje en el proceso de la autorregulación (ver p. ej., Hill y Hannafin, 1997).

5) El estudio de la influencia del desarrollo evolutivo en el proceso de la autorregulación del aprendizaje. Por ejemplo, Pintrich y Zusho (2002) han revisado los estudios centrados en el análisis de los efectos de distintas variables motivacionales (autoeficacia, metas, valor e interés por la tarea) y cognitivas (p. ej., conocimiento metacognitivo, conocimiento previo, memoria de trabajo) en el aprendizaje autorregulado, destacando el papel mediador del desarrollo evolutivo (edad) en la naturaleza de dicha relación.

6) El examen del papel que tienen las diferencias individuales y culturales en el aprendizaje autorregulado. En relación con el primer aspecto, se podría estudiar, siguiendo a Paris y Paris (2001, p. 99), la influencia del temperamento, la impulsividad, la paciencia o la resistencia ante las distracciones en el proceso de la autorregulación. Asimismo, el estudio de las diferencias de género sigue siendo una cuestión candente dentro de la investigación psicoeducativa. Si bien, la investigación empírica muestra que podrían existir diferencias en el uso de ciertas estrategias de autorregulación a favor de las chicas (p. ej., Ablard y Lipschultz, 1998), sin embargo, los hallazgos obtenidos han sido atribuidos a sesgos en las respuestas de los chicos y las chicas a los cuestionarios de autoinforme más que a la existencia de diferencias re- 
ales (cfr. Pintrich y Zusho, 2002, p. 276). Por otro lado, una cuestión de gran interés es saber si los hallazgos a los que ha llegado la investigación sobre aprendizaje autorregulado, en la que se ha utilizado principalmente alumnos de culturas occidentales (EE UU, Europa, Canadá), se pueden generalizar a sujetos de otras culturas.

7) La enseñanza de los diferentes procesos que intervienen en el aprendizaje autorregulado dentro de las diferentes áreas del currículo. Como señalan Schunk y Zimmerman (2003, p. 74; ver también Schunk y Ertmer, 2000), es importante integrar las actividades y estrategias de autorregulación dentro del contexto escolar y de las diferentes materias curriculares, y ayudar a los alumnos a modificarlas y adaptarlas ante distintas situaciones de aprendizaje.

\section{Conclusiones}

Actualmente, el estudio de qué es el aprendizaje autorregulado, qué procesos y estrategias están implicadas en él y cómo enseñarlas se ha convertido en una cuestión candente dentro de la Psicología de la Educación y en una de las principales direcciones por donde está avanzando esta disciplina.

El aprendizaje autorregulado es una fusión de skill y will, de destreza y de voluntad. El aprendiz estratégico es aquel que ha aprendido a planificar, controlar y evaluar sus procesos cognitivos, motivacionales/afectivos, comportamentales y contextuales. Sabe cómo aprende, está automotivado, conoce sus posibilidades y limitaciones, y en función de ese conocimiento, controla y regula sus procesos de aprendizaje para adecuarlos a los objetivos de las tareas y al contexto, optimizar su rendimiento y mejorar sus habilidades mediante la práctica.

Una de las características de los alumnos que autorregulan su aprendizaje es el control de su motivación y de sus emociones. En concreto, la investigación destaca el papel de las percepciones de autoeficacia y de las metas. Con respecto a estas últimas, en un primer momento, la investigación puso de relieve que los alumnos orientados hacia metas de rendimiento (demostrar competencia) mostraban un patrón motivacional, cognitivo y comportamental perjudicial para el aprendizaje y el rendimiento. Sin embargo, en los últimos años, a partir de la reconceptualización de la teoría de metas llevada a cabo por Pintrich, Barron, Elliot y 
Harackiewicz se destaca los efectos positivos de estas metas en la motivación y en el rendimiento, y los beneficios de perseguir múltiples metas al mismo tiempo.

Hasta la fecha, los cuestionarios de autoinforme han sido los instrumentos más utilizados para evaluar los distintos procesos implicados en el aprendizaje autorregulado. No obstante, la investigación actual, basada en una perspectiva socioconstructivista, está empezando a utilizar cada vez más instrumentos introspectivos y cualitativos con el objetivo de capturar la naturaleza dinámica, procesual y social de la autorregulación.

En los últimos años hemos asistido a un cambio muy importante en el área de la intervención. Actualmente, los modelos instruccionales destacan la importancia de la práctica autorreflexiva, el aprendizaje colaborativo y el scaffolding (andamiaje) en la enseñanza de la autorregulación. Asimismo, se enfatiza que la intervención se centre en ambientes naturales, utilizando tareas auténticas y contextualizadas, unidas a los intereses y necesidades de los alumnos, ya que ello les va a permitir generalizar lo aprendido a situaciones de su vida personal, académica y social.

El papel del contexto, el perfeccionamiento de la metodología de la investigación y de los instrumentos de medida, la enseñanza de las estrategias de autorregulación dentro del currículo, la influencia del desarrollo evolutivo en la autorregulación y el papel de ciertas variables intra e interpersonales son algunas de las futuras direcciones por donde puede avanzar la investigación en este campo. Un campo que está ayudando a comprender mejor los factores implicados en el proceso de enseñanza-aprendizaje, y a desarrollar propuestas de intervención dirigidas a paliar las dificultades de los alumnos, originadas por su falta de conciencia y control sobre el aprendizaje, y optimizar su rendimiento académico.

\section{Referencias}

Ablard, K.E. y Lipschultz, R.E. (1998). Self-regulated learning in high achieving students: Relations to advances reasoning, achievement goals, and gender. Journal of Educational Psychology, 90, 94-101.

Ames, C. (1992). Classrooms: Goals, structures, and student motivation. Journal of Educational Psychology, 84, 261-271. 
Baker, L. y Cerro, L.C. (2000). Assessing metacognition in children and adults. En G. Schraw y J.C. Ampara (Eds.), Issues in the measurement of metacognition (pp. 99-145). Lincoln: Buros Institute of Mental Measurements, University of Nebraska Press.

Bandura, A. (1977). Self-efficacy: Toward a unifying theory of behavioral change. Psychological Review, 84, 191-215.

Bandura, A. (1986). Social foundations of thought and action. Englewood Cliffs, NJ: Prentice Hall. (Trad. cast: Pensamiento y acción. Fundamentos sociales. Barcelona: Martínez Roca, 1987).

Bandura, A. (1997). Self-efficacy: The exercise of control. New York: Freeman.

Bandura, A. (2001). Social cognitive theory: An agentic perspective. Annual Review Psychology, 52, 1-26.

Barron, K.E. y Harackiewicz, J.M. (2000). Achievement goals and optimal motivation: A multiple goals approach. En C. Sansone y J. Harackiewicz (Eds.), Intrinsic and extrinsic motivation (pp. 229-254). San Diego, CA: Academic Press.

Beltrán, J. (1996). Concepto, desarrollo y tendencias actuales de la Psicología de la Instrucción. En J. Beltrán y C. Genovard (Eds.), Psicología de la instrucción: variables y procesos básicos (Vol. 1, pp. 19-86). Madrid: Síntesis/Psicología.

Blumenfeld, P.C. y Marx, R. . (1997). Motivation and cognition. En H.J. Walberg y G.D. Haertel (Eds.), Psychology and educational practice (pp. 79-106). Berkeley, CA: McCutchan.

Boekaerts, M., Pintrich, P.R. y Zeidner, M. (2000). Handbook of self-regulation. San Diego, CA: Academic Press.

Bong, M. (1998). Tests of the internal/external frames of reference model with subjectspecific academic self-efficacy and frame-specific academic self-concepts. Journal of Educational Psychology, 90, 102-110.

Bong, M. y Clark, R.E. (1999). Comparison between self-concept and self-efficacy in academic motivation research. Educational Psychologist, 34, 139-154.

Bong, M. y Skaalvik, E. (2003). Academic self-concept and self-efficacy: How different are they really? Educational Psychology Review, 15, 1-40.

Brown, A.L. (1997). Transforming schools into communities of thinking and learning about serious matters. American Psychologist, 52, 399-413.

Brown, A.L. y Campione, J.C. (1990). Communities of learning and thinking or a context by any other name. Human Development, 21, 108-125. 
Butler, D.L. (2002). Qualitative approaches to investigating self-regulated learning: Contributions and challenges. Educational Psychologist, 37, 59-63.

Butler, D.L. y Winne, P. H. (1995). Feedback and self-regulated learning: A theoretical synthesis. Review of Educational Research, 65, 245-28.

Corno, L. (1993). The best-laid plans: Modern conceptions of volition and educational research. Educational Researcher, 22, 14-22.

Corno, L. (2001). Volitional aspects of self-regulated learning. En B. J. Zimmerman y D. H. Schunk (Eds.), Self-regulated learning and academic achievement: Theoretical perspectives (pp. 191-225). Hillsdale, NJ: Erlbaum.

Deci, E.L. y Ryan, R.M. (2002). Handbook of-self-determination research. New York: University of Rochester Press.

De la Fuente, J. y Martínez Vicente, J.M. (2000). PRO\&REGULA: un programa para aprender a autorregularse durante el aprendizaje (Vol. I y II). Málaga: Aljibe.

Durán, M.L. (1999). Estrategias de aprendizaje y asesoramiento académico en el primer año de universidad. Tesis doctoral no publicada. Pamplona: Universidad de Navarra.

Dweck, C.S. (1986). Motivational processes affecting learning. American Psychologist, 41, 1040-1048.

Dweck, C.S. (2002). Messages that motivate: How praise molds students' beliefs, motivation, and performance (in surprising ways). En J. Aronson (Ed.), Improving academic achievement: Impact of psychological factors on education (pp. 37-60). San Diego, CA: Academic Press.

Eccles, J.S., Wigfield, A. y Schiefele, U. (1998). Motivation to succeed. En W. Damon y N. Eisenberg (Eds.), Handbook of child psychology: Social, emotional, and personality development (5th ed., Vol. 3. pp. 1017-1095). New York: Wiley.

Elliot, A.J. (1999). Approach and avoidance motivation and achievement goals. Educational Psychologist, 34, 169-189.

García Ros, R., Clemente, A. y Pérez González, F. (1994). Intervención escolar en estrategias de aprendizaje. Valencia: CSV.

Garner, R. (1987). Metacognition and reading comprehension. Norwood, NJ: Ablex.

González Pienda, J.A., Núñez, J.C., Rodríguez, S. y González Cabanach, R. (1994, Sept.). Evaluación de estrategias de estudio y aprendizaje escolar. IV Congreso de Evaluación Psicológica. Santiago de Compostela.

González Torres, M.C. (1997). La motivación académica. Pamplona: EUNSA. 
González Torres, M.C. (2003, Marzo). De los deseos a la acción: estrategias para favorecer la automotivación de los alumnos. Ponencia presentada en las V Jornadas de Innovación Pedagógica. Attendis Colegios, Algeciras.

González Torres, M.C. y Tourón, J. (1992). Autoconcepto y rendimiento escolar. Sus implicaciones en la motivación y en la autorregulación del aprendizaje. Pamplona: EUNSA.

Graham, S., Harris, K.R. y Troia, G.A. (1998). Writing and self-regulation: Cases from the self-regulated strategy development model. En D.H. Schunk y B.J. Zimmerman (Eds.), Self-regulated learning: From teaching to self-reflective practice (pp. 20-41). New York: Guilford.

Harackiewicz, J.M., Barron, K.E., Pintrich, P.R., Elliot, A.E. y Thrash, T.M. (2002). Revision of achievement goal theory: Necessary and illuminating. Journal of Educational Psychology, 94, 638-645.

Heckhausen, H. (1991). Motivation and action. Berlín: Springer-Verlag.

Hernández, P. y García, L.A. (1997). Enseñar a pensar. Un reto para los profesores. Notice. La Laguna: Tafor.

Hill, J.R. y Hannafin, M.J. (1997). Cognitive strategies and learning from the world wide web. Educational Techonology Research and Development, 45, 37-64.

Kaplan, A., Middleton, M.J., Urdan, T. y Midgley, C. (2002). Achievement goals and goal structures. En C. Midgley (Ed.), Goals, goal structure and pattern of adaptative learning (pp. 21-53). Mahwah, NJ: Erlbaum.

Kuhl, J. (2000). A functional-design approach to motivation and self-regulation. En M. Boekaerts, P. R. Pintrich y M. Zeidner (Eds.), Handbook of self-regulation (pp. 111-169). San Diego, CA: Academic Press.

Ley, K. y Young, D.B. (2001). Instructional principles for self-regulation. Educational Technology, Research and Development, 49, 93-104.

Lin, Y.G., McKeachie, W.J. y Kim, Y.C. (2003). College student intrinsic and/or extrinsic motivation and learning. Learning and Individual Differences, 13, 251-258.

Marsh, H.W. (1990). The structure of academic self-concept: The Marsh/Shavelson Model. Journal of Educational Psychology, 82, 623-636.

McCaslin, M. y Hickey, D.T. (2001). Educational psychology, social constructivism, and educational practice: A case for emergent identity. Educational Psychologist, 36, 133140.

McCombs, B.J. y Marzano, R.J. (1990). Putting the self-regulated learning: The self as agent in integrating will and skill. Educational Psychologist, 25, 51-69. 
McCombs, B.L. y Whisler, J.S. (1997). The learner-centered classroom and school. San Francisco, CA: Jossey-Bass (Trad. cast: La clase y la escuela centradas en el aprendiz. Barcelona: Paidós. 2000).

McCombs, B. L. y Whisler, J. S. (2000). La clase y la escuela centrada en el aprendiz. Barcelona: Paidós.

Middleton, M.J. y Midgley, C. (1997). Avoiding the demonstration of lack of ability: An underexplored aspect of goal theory. Journal of Educational Psychology, 89, 710-718.

Midgley, C. (Ed.). (2002). Goals, goal structure and pattern of adaptative learning. Mahwah, NJ: Erlbaum.

Midgley, C., Middleton, M. y Kaplan, A. (2001). Performance-approach goals: Good for what, for whom, under what circumstances, and what cost? Journal of Educational Psychology, 93, 77-86.

Midgley, C. y Urdan, T. (2001). Academic self-handicapping and achievement goals: A further examination. Contemporary Educational Psychology, 26, 61-75.

Monereo, C. y Castelló, M.B. (1997). Las estrategias de aprendizaje: cómo incorporarlas a la práctica educativa. Barcelona: Edebé.

Nelson, T. y Narens, L. (1990). Metamemory: A theoretical framework and new findings. En G. Boewr (Ed.), The psychology of learning and motivation (Vol. 26, pp. 125-141). New York: Academic Press.

Newman, R. (1998). Students' help-seeking during problem solving: Influences of personal and contextual goals. Journal of Educational Psychology, 90, 644-658.

Nicholls, J.G. (1984). Conceptions of ability and achievement motivation. En R. Ames y C. Ames (Eds.), Research on motivation and education: Student motivation (Vol. 1, pp. 39-73). San Diego, CA: Academic Press.

Niemivirta, M. (1998). Individual differences in motivational and cognitive factors affecting self-regulated learning: A pattern-oriented approach. In P. Nenniger, R.S. Jager y M. Wosnitza (Eds.), Advances in motivation (pp. 32-42). Landau: Verlag Empirische Padagogik.

Onrubia, J. (1996). Mediación y construcción de significados en la interacción profesor/alumnos y en la interacción entre alumnos. En A. Barca, J.A. González Pienda, R. González Cabanach y J. Escoriza (Eds.), Psicología de la instrucción: componentes contextuales y relacionales del aprendizaje escolar (Vol. 3, pp. 21-43). Barcelona: EUB.

Pajares, F. y Schunk, D.H. (2001). Self-beliefs and school sucess: Self-efficacy, self-concept, 
and school achievement. En R. Riding y S.G. Rayner (Eds.), International perspectives on individual differences: Self-perception (Vol. 2, pp. 239-265). Westport, CT: Ablex.

Pajares, F. y Schunk, D.H. (2002). Self and self-belief in psychology and education: A historical perspective. En J. Aronson (Ed.), Improving academic achievement: Impact of psychological factors on education (pp. 3-21). San Diego, CA: Academic Press.

Paris, S.G., Byrnes, J.P. y Paris, A.H. (2001). Constructing theories, identities, and actions of self-regulated learners. En B.J. Zimmerman y D.H. Schunk (Eds.), Self-regulated learning and academic achievement: Theoretical perspectives (pp. 253-287). Hillsdale, NJ: Erlbaum.

Paris, S.G. y Paris, A.H. (2001). Classroom applications of research on self-regulated learning. Educational Psychologist, 36, 89-101.

Patrick, H., Anderman, L.H. y Ryan, A.M. (2002). Social motivation and the classroom social environment. En C. Midgley (Ed.), Goals, goal structure and pattern of adaptative learning (pp. 85-108). Mahwah, NJ: Erlbaum.

Patrick, H. y Middleton, M.J. (2002). Turning the kaleidoscope: What we see when selfregulated learning is viewed with a qualitative lens. Educational Psychologist, 37, 27 39.

Perry, N.E. (1998). Young children's self-regulated learning and contexts that support it. Journal of Educational Psychology, 90, 715-729.

Pintrich, P.R. (2000a). Educational Psychology at the millennium: A look back and a look forward. Educational Psychologist, 35, 221-226.

Pintrich, P.R. (2000b). The role of goal orientation in self-regulated learning. En M. Boekaerts, P.R. Pintrich y M. Zeidner (Eds.), Handbook of self-regulation (pp. 451502). San Diego, CA: Academic Press.

Pintrich, P.R. (2000c). Multiple goals, multiple pathways: The role of goal orientation in learning and achievement. Journal of Educational Psychology, 92, 544-555.

Pintrich, P.R. (2003). Motivation and classroom learning. En W.M. Reynolds y G.E. Miller (Eds.), Handbook of psychology: Educational psychology (Vol. 7, pp. 103-122). Hoboken, NJ: Wiley.

Pintrich, P.R., Smith, D., García, T. y McKeachie, W.J. (1991). A manual for the use of the Motivated Strategies for Learning Questionnaire (MSLQ). Ann Arbor: University of Michigan, School of Education. 
Pintrich, P.R., Wolters, C. y Baxter, G.P. (2000). Assessing metacognition and self-regulated learning. En G. Schraw y J. C. Ampara (Eds.), Issues in the measurement of metacognition (pp. 43-97). Lincoln: Buros Institute of Mental Measurements, University of Nebraska Press.

Pintrich, P.R. y Zusho, A. (2002). The development of academic self-regulation: The role of cognitive and motivational factors. En A. Wigfield y J. S. Eccles (Eds.), Development of achievement motivation (pp. 249-284). San Diego, CA: Academic Press.

Pressley, M. (2000). Development of grounded theories of complex cognitive processing: Exhaustive within-and between-study analyses of think-aloud data. En G. Schraw y J. C. Impara (Eds.), Issues in the measurement of metacognition (pp. 261-296). Lincoln: Buros Institute of Mental Measurements, University of Nebraska Press.

Pressley, M. y Afflerbach, P. (1995). Verbal protocols of reading: The nature of constructively responsive reading. Hillsdale, $\mathrm{NJ}$ : Erlbaum.

Prieto, M.D. y Castejón, J.L. (1993, Mayo). El LASSI: una escala para evaluar estrategias de aprendizaje. Comunicación presentada al III Congreso INFAD. León.

Puustinen, M. y Pulkkinen, L. (2001). Models of self-regulated learning: A review. Scandinavian Journal of Educational Research, 45, 269-286.

Reyero, M. y Tourón, J. (2003). El desarrollo del talento: la aceleración como estrategia educativa. A Coruña: Netbiblo.

Reynolds, W.M. y Miller, G.E. (2003). Current perspectives in educational psychology. En W.M. Reynolds y G.E. Miller (Eds.), Handbook of psychology: Educational psychology (Vol. 7, pp. 3-20). Hoboken, NJ: Wiley.

Roces, C. (1996). Estrategias de aprendizaje y motivación en la universidad. Tesis doctoral no publicada. Pamplona: Universidad de Navarra.

Roces, C. y González Torres, M.C. (1998). Capacidad de autorregulación del aprendizaje. En J.A. González Pienda y J.C. Núñez (Eds.), Dificultades de aprendizaje escolar (pp. 239-259). Madrid: Pirámide/Psicología.

Roces, C., Tourón, J. y González Torres, M.C. (1995). Validación preliminar del CEAM II. Psicológica, 16, 347-366.

Rodríguez, S. (1999). Autorregulación motivacional del aprendizaje: características diferenciales e implicaciones cognitivo-motivacionales. Tesis doctoral no publicada. España: Universidad de la Coruña. 
Ryan, A.M. y Pintrich, P.R. (1997). Should I ask for help?: The role of motivation and attitudes in adolescent's help-seeking in math class. Journal of Educational Psychology, 89, $329-341$.

Sanz de Acedo, M.L. e Iriarte, M.D. (1999). Cognición en el aula. Teoría y práctica Pamplona: Universidad Pública de Navarra.

Schraw, G. y Impara, J.C. (Eds.). (2000). Issues in the measurement of metacognition. Lincoln: Buros Institute of Mental Measurements, University of Nebraska Press.

Schunk, D.H. (2001). Social cognitive theory and self-regulated learning. En B.J. Zimmerman y D.H. Schunk (Eds.), Self-regulated learning and academic achievement: Theoretical perspectives (pp. 125-151). Hillsdale, NJ: Erlbaum.

Schunk, D.H. y Ertmer, P.A. (2000). Self-regulation and academic learning: Self-efficacy enhancing interventions. En M. Boekaerts, P. R. Pintrich y M. Zeidner (Eds.), Handbook of self-regulation (pp. 631-647). San Diego, CA: Academic Press.

Schunk, D.H. y Zimmerman, B.J. (1994). Self-regulation of learning and perfomance: Issues and educational applications. Hillsdale, NJ: Erlbaum.

Schunk, D.H. y Zimmerman, B.J. (1998). Self-regulated learning: From teaching to selfreflective practice. New York: Guilford.

Schunk, D.H. y Zimmerman, B.J. (2003). Self-regulation and learning. En W.M. Reynolds y G.E. Miller (Eds.), Handbook of psychology: Educational psychology (Vol. 7, pp. 5978). Hoboken, NJ: Wiley.

Shavelson, R.J., Hubner, J.J. y Stanton, G.C. (1976). Validation of constructs interpretations. Review of Educational Research, 46, 407-441.

Skaalvik, E.M. (1997). Issues in research on self-concept. En M.L. Maehr y P.R. Pintrich (Eds.), Advances in motivation and achievement (Vol. 10, pp. 51-97). Greenwich, CT: JAI Press.

Turner, J.C. (1995). The influence of classroom contexts on young children's motivation for literacy. Reading Research Quarterly, 30, 410-441.

Urdan, T.C. (1997). Achievement goal theory: Past results, future directions. En M.L. Maehr y P.R. Pintrich (Eds.), Advances in motivation and achievement (Vol. 10, pp. 99-141). Greenwich, CO: JAI Press.

Urdan, T.C. y Maehr, M.L. (1995). Beyond a two-goal theory of motivation and achievement: A case for social goals. Review of Educational Research, 65, 213-243. 
Urdan, T., Ryan, A. M., Anderman, E.M. y Gheen, M.H. (2002). Goals, goal structures, and avoidance behaviors. En C. Midgley (Ed.), Goals, goal structure and pattern of adaptative learning (pp. 55-83). Mahwah, NJ: Erlbaum.

Valle, A., González Cabanach, R., Núñez, J.C., González Pienda., Rodríguez, S. y Piñeiro, I. (2003). Multiple goals, motivation, and academic learning. British Journal of Educational Psychology, 73, 71-87.

Valle, A., González Cabanach, R., Vieiro, P. y Suárez, J.M. (1998). Las estrategias de aprendizaje. En A. Valle y R. González Cabanach (Eds.), Psicología de la educación, I: variables personales y aprendizaje escolar (pp. 183-213). La Coruña: Universidade Da Coruña.

Volet, S. y Jarvela, S. (Eds.) (2001). Motivation in learning contexts: Theoretical advances and methodological implications. Amsterdam: Elsevier.

Weinstein, C.E., Husman, J. y Dierking, D.R. (2000). Self-regulation interventions with a focus on learning strategies. En M. Boekaerts, P. R. Pintrich, y M. Zeidner (Eds.), Handbook of self-regulation (pp. 728-748). San Diego, CA: Academic Press.

Weinstein, C.E., Schulte, A.C. y Palmer, D.R. (1987). LASSI: Learning and study strategies inventory. Clearwater, FL: H. Y H. Publishing.

Wentzel, K.R. (2000). What is it that I'm trying to achieve? Classroom goals from a content perspective. Contemporary Educational Psychology, 25, 105-115.

Wentzel, K.R. (2002). The contribution of social goal setting to children's school adjustment. En A. Wigfield y J.S. Eccles (Eds.), Development of achievement motivation (pp. 221246). San Diego, CA: Academic Press.

Wentzel, K.R. (2003). School adjustment. En W. M. Reynolds y G. E. Miller (Eds.), Handbook of psychology: Educational psychology (Vol. 7, pp. 235-258). Hoboken, NJ: Wiley.

Wigfield, A. y Eccles, J.S. (2000). Expectancy-value theory of achievement motivation. Contemporary Educational Psychology, 25, 68-81.

Winne, P.H. (1995). Inherent details in self-regulated learning. Educational Psychologist, 30, 173-187.

Winne, P.H. y Jamieson-Noel, D. (2003). Self-regulating studying by objectives for learning: Students' reports compared to a model. Contemporary Educational Psychology, 28, 259-276.

Winne, P.H., Jamieson-Noel, D. y Muis, K.R. (2002). Methodological issues and advances in researching tactics, strategies, and self-regulated learning. En P. R. Pintrich y M. L. 
Maehr (Eds.), Advances in motivation and achievement: New directions in measures and methods (Vol. 12, pp. 121-155). Greenwich, CO: JAI Press.

Winne, P.H. y Perry, N.E. (2000). Measuring self-regulated learning. En M. Boekaerts, P. R. Pintrich, y M. Zeidner (Eds.), Handbook of self-regulation (pp. 531-566). San Diego, CA: Academic Press.

Wolters, C.A. (2003). Understanding procrastination from a self-regulated learning perspective. Journal of Educational Psychology, 95, 179-187.

Yuste, C. y Ayala, C. (2000). Programas para la estimulación de las habilidades básicas de la inteligencia: estrategias de regulación y motivación para aprender (PROGRESINT) (Vol. 31). Madrid: CEPE.

Zeidner, M., Boekaerts, M. y Pintrich, P.R. (2000). Self-regulation: Directions and challenges for future research. En M. Boekaerts, P.R. Pintrich y M. Zeidner (Eds.), Handbook of self-regulation (pp. 749-768). San Diego, CA: Academic Press.

Zimmerman, B.J. (1998). Developing self-fulfilling cycles of academic regulation: An analysis of exemplary instructional model. En D. H. Schunk y B. J. Zimmerman (Eds.), Self-regulated learning: From teaching to self-reflective pratice (pp. 1-19). New York: Guilford.

Zimmerman, B.J. (2000). Attaining self-regulation: A social cognitive perspective. En M. Boekaerts, P. R. Pintrich y M. Zeidner (Eds.), Handbook of self-regulation (pp. 451502). San Diego, CA: Academic Press.

Zimmerman, B.J. (2001). Achieving academic excellence: A self-regulatory perspective. En M. Ferrari (Ed.), The pursuit of excellence through education (pp. 85-110). Mahwah, NJ: Erlbaum.

Zimmerman, B.J. (2002). Becoming self-regulated learned: An overview. Theory into Practice, 41, 64-72.

Zimmerman, B.J. y Martínez-Pons, M. (1986). Development of a structured interview for assessing student use of self-regulated learning strategies. American Educational Research Journal, 23, 614-628.

Zimmerman, B.J. y Martínez-Pons, M. (1988). Construct validation of a strategy model of student self-regulated learning. Journal of Educational Psychology, 80, 284-190.

Zimmerman, B.J. y Schunk, D.H. (Eds.). (1989). Self-regulated learning and academic achievement: Theory, research and practice. New York: Springer-Verlag.

Zimmerman, B.J. y Schunk, D.H. (Eds.). (2001). Self-regulated learning and academic achievement: Theoretical perspectives. Hillsdale, NJ: Erlbaum. 
El aprendizaje autorregulado: presente y futuro de la investigación

[Página dejada en blanco intencionadamente] 